\title{
The velocity structure of Cygnus OB2
}

\author{
Becky Arnold, ${ }^{1,2 \star ~ S i m o n ~ P . ~ G o o d w i n ~}{ }^{1}$ and Nick J. Wright ${ }^{3}$ \\ ${ }^{1}$ Department of Physics and Astronomy, University of Sheffield, Sheffield S3 7RH, UK \\ ${ }^{2}$ Department of Astronomy, University of Massachusetts, Massachusetts, 01003, USA \\ ${ }^{3}$ Astrophysics Group, Keele University, Keele, Staffordshire, ST5 5BG, UK
}

Accepted XXX. Received YYY; in original form ZZZ

\begin{abstract}
The kinematic structure of the Cygnus OB2 association is investigated. No evidence of expansion or contraction is found at any scale within the region. Stars that are within $\sim 0.5$ parsecs of one another are found to have more similar velocities than would be expected by random chance, and so it is concluded that velocity substructure exists on these scales. At larger scales velocity substructure is not found. We suggest that bound substructures exist on scales of $\sim 0.5$ parsecs, despite the region as a whole being unbound. We further suggest that any velocity substructure that existed on scales $>0.5$ parsecs has been erased. The results of this study are then compared to those of other kinematic studies of Cygnus OB2.
\end{abstract}

Key words: stars: kinematics and dynamics - open clusters and associations: general - stars: formation

\section{INTRODUCTION}

Star forming regions are the focus of a great deal of scientific interest, and for good reason. They inform our understanding of how stars are born and how their environments evolve. Their study is also vital for our comprehension of the conditions that planets form in, and the type/number of planets which may exist in the universe.

Spatial and dynamical structure are perhaps the most important aspects defining a star forming region, but can be difficult to interpret. A number of statistical methods have been developed to quantify different aspects of the spatial structure of these regions (Allison et al. 2009; Cartwright 2009; Maschberger \& Clarke 2011; Buckner et al. 2019), but they don't touch upon its velocity structure. However the Velocity Structure Analysis Tool (VSAT) (Arnold \& Goodwin 2019) does, and it is used in this paper to investigate the velocity structure of Cygnus OB2, which has previously had relatively little statistical kinematic analysis.

Cygnus OB2 lies at a distance of approximately 1400 parsecs (Hanson 2003; Rygl et al. 2012; Berlanas et al. 2019), and has an estimated stellar mass of order $10^{4} M_{\odot}$ (Drew et al. 2008; Wright et al. 2010). Estimates of the region's age vary, for example Massey et al. (1995) find an age of 1-3 Myr and Wright et al. (2015) find an age up up to $7 \mathrm{Myr}$ with star formation peaking 4-5 Myr ago. Given a number of estimates it seems relatively certain that the age of the region lies somewhere between 1 and $7 \mathrm{Myr}$.

This region is chosen because it has been extensively

^ rebeccajasmi@umass.edu studied, (Massey \& Thompson 1991; Knödlseder 2000; Comerón et al. 2008; Kiminki et al. 2015; Roquette et al. 2017; Berlanas et al. 2018, 2019) meaning there is a large amount of observational data already available. There have also been studies focused on its spatial and kinematic structure (Wright et al. 2014, 2016; Winter et al. 2019). This is useful as it allows findings relating to the kinematic structure of the region which are achieved using different techniques to be compared to see if they are consistent.

The structure of this paper is as follows. In section 2 the methods used to collect and analyse the data are outlined. In section 3 the results of the analysis are reported and in section 4 they are discussed. Finally in section 5 the conclusions drawn from the results are summarised.

\section{METHODS}

\subsection{Data collection}

For this work we use the X-ray selected sample of Cygnus OB2 members presented by Wright \& Drake (2009) for the central portion of the association. X-rays provide a largely unbiased diagnostic of youth that is effective for separating young association members from older field stars, and Wright et al. (2010) made further efforts to identify and remove foreground contaminants from the sample. Proper motions for these stars were derived by Wright et al. (2016) as part of the DANCe (Dynamical Analysis of Nearby Clusters) project (Bouy et al. 2013).

The sample includes many of the known high-mass Otype stars in Cygnus OB2 with masses up to $100 M_{\odot}$, as well 
as low-mass stars down to $0.1 M_{\odot}$. The sample is estimated to be mostly complete to $\sim 0.8 M_{\odot}$ (Wright et al. 2014). In contrast only around 70 per cent of these sources appear in the Gaia survey results and approximately half of those do not survive Gaia's recommended astrometric quality cuts (Arenou et al. 2018; Lindegren 2018; Lindegren et al. 2018). This is because the sources are mostly fainter than is optimal for detection by Gaia.

The dataset used in this paper can be seen in Fig. 1. In this figure each star is shown as a dot with a line. The location of the dot indicates the location of the star and the length and direction of the line indicates the star's velocity. In the top right of the figure a velocity vector of 10 mas $\mathrm{yr}^{-1}$ is shown with the median velocity uncertainty of the dataset shown by a grey cone. This is done to give a visual representation of the uncertainties on the velocity data.

Visual inspection of the figure shows a small number of stars with velocities far greater than most. To investigate this we conduct an exploratory analysis of the data. For additional details relating to the origins of this dataset see Wright et al. (2016).

\subsection{Exploratory analysis}

Fig. 2 shows a scatter plot of the stellar velocities in the reference frame of the region. As was the case for Fig. 1 it is visually apparent there are a small number of outliers. Additionally Fig. 3 shows a histogram with the stars binned by the number of standard deviations their speed is from the mean stellar speed of the dataset, and from this it is clear there is a small number of stars which have speeds many standard deviations in excess of the mean. Note that in Fig. 3 the $y$-axis is logarithmic in order to make bins with few entries more easily visible.

The presence of extreme velocity outliers is also noted in Wright et al. (2016) where this dataset was first presented. That work concludes that these outliers are most likely due to:

- background/foreground stars that have been mistaken for members of Cygnus OB2;

- stars ejected from Cygnus OB2 by dynamical events such as the disruption of binary systems;

- stars now in the region of Cygnus OB2 but originating in nearby star clusters/associations which dispersed.

Regardless of their origin it is clear there is a small number of the 873 stars with very different velocities to the rest of the sample. Further, it seems unlikely that they are representative of the region's underlying velocity structure. Due to this, stars with speeds more than three standard deviations from the mean stellar speed in the dataset (a total of eight stars) are removed. This removal has very little impact on the results, and the effects it does have are discussed later. The removed stars are shown in grey in Fig. 1 and outside the grey inset in Fig. 2

In Fig. 2 velocities appear to be centrally concentrated but, particularly in the inset, there is no further obvious velocity structure apparent. To further investigate the velocity structure we plot stellar speeds against their distances from the centre, see Fig. 4. The centre is defined here as the mean position of the stars.

There appears to be a peak in this distribution of points towards the middle of the plot but it is difficult to assess meaningfully by eye. To better understand it the moving median is calculated and is shown by a black line on Fig. 4 . Upon close examination the moving median appears slightly elevated at distances between $\sim 2$ and $3 \mathrm{pc}$, indicating stars with high speeds are preferentially located at moderate distances from the centre of the field of view. That said, this effect is extremely slight and the function is largely flat indicating that any relationship between speed and position is weak.

\subsection{Data analysis}

The VSAT method (Arnold \& Goodwin 2019) is applied to analyse the velocity structure of the dataset in more detail. In brief the method is as follows: for every possible pair of stars this method calculates the distance between them $(\Delta r)$ and their velocity difference $(\Delta v)$. To clarify, here a 'pair of stars' does not necessarily refer to a binary system, but just to any two stars in the region. The pairs are then binned by $\Delta r$ and within each bin the average $\Delta v$ is calculated. Finally these are plotted against each other. The uncertainties on the velocities are propagated when calculating each $\Delta v$, and these errors are used to weight the average. The impact of stochasticity on these uncertainties is also incorporated, see Arnold \& Goodwin (2019) for full details of how these calculations are performed.

The VSAT method is applied twice, each time using a different definition of the velocity difference between two stars $\Delta v$. The first case is referred to as the magnitude definition, $\Delta v_{\mathrm{M}}$, and it is defined as the magnitude of the difference between the two star's velocity vectors. Therefore for stars $a$ and $b \Delta v_{\mathrm{M}}$ is calculated as:

$$
\Delta v_{a b \mathrm{M}}=\left|\mathbf{v}_{a}-\mathbf{v}_{b}\right|
$$

This definition is particularly useful as a raw measure of how similar/different stellar velocity vectors are.

The second way $\Delta v$ is defined is as the time differential of $\Delta r$, i.e. the rate at which the distance between the stars is changing. This is referred to as the directional definition, $\Delta v_{\mathrm{D}}$. For stars $a$ and $b$ this is

$\Delta v_{a b \mathrm{D}}=\frac{\left(\mathbf{r}_{a}-\mathbf{r}_{b}\right) \cdot\left(\mathbf{v}_{a}-\mathbf{v}_{b}\right)}{\Delta r_{a b}}$.

In this definition if the stars are moving away from each other $\Delta r$ increases so $\Delta v_{\mathrm{D}}$ is positive, and if the stars are moving towards each other $\Delta r$ decreases and $\Delta v_{\mathrm{D}}$ is negative. This definition is particularly useful for studying if regions or sub-regions are undergoing expansion/collapse. It as also useful for studying the relative motions of different substructures within a region. Both of these things are helpful in discerning the dynamical state of a region and its history.

In order to facilitate the calculation of these parameters the data is converted from RA, Dec, and proper motions into a Cartesian coordinate scheme. The SKYCOORD class in the Python package Astropy is used to perform this conversion. 


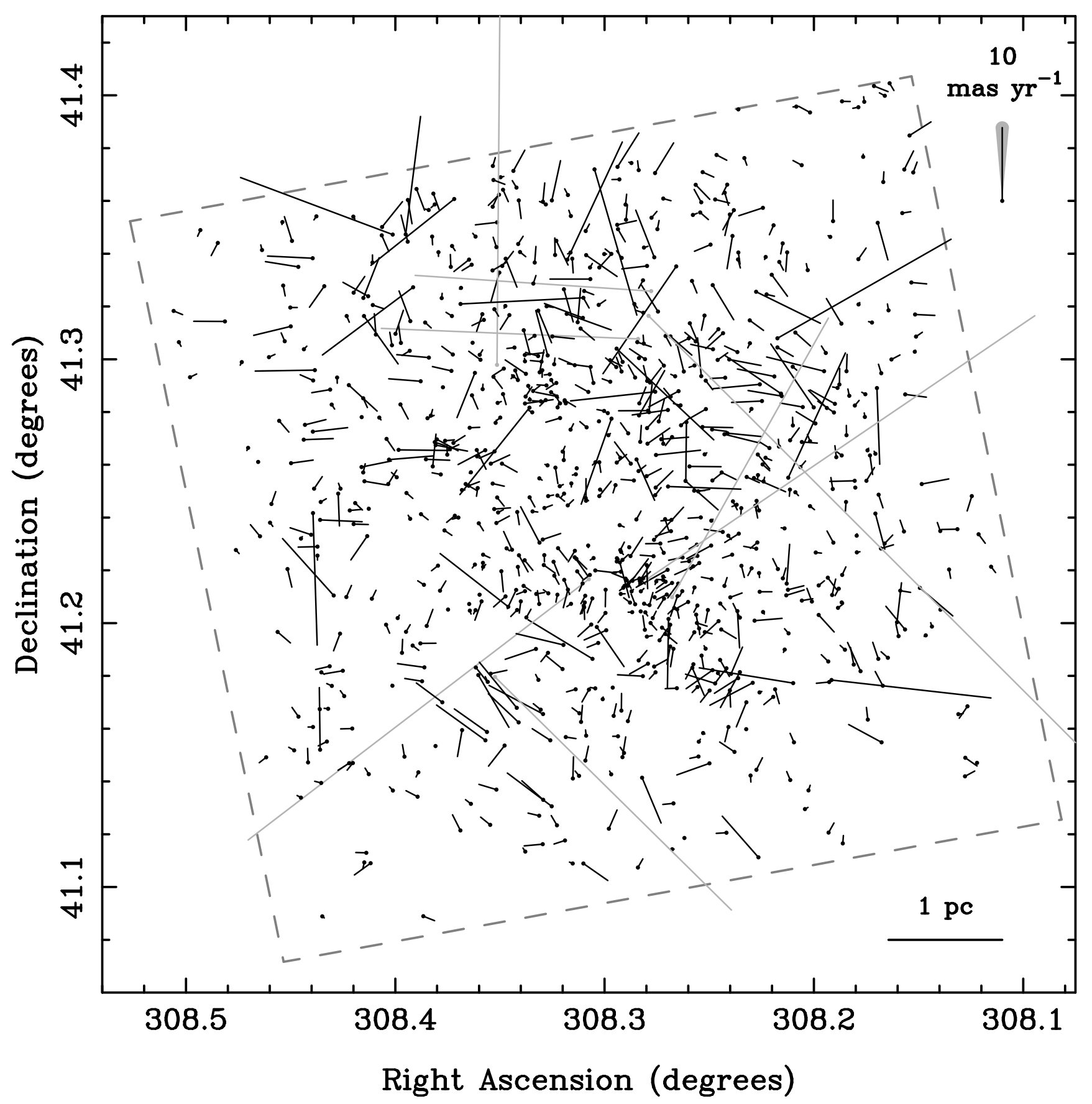

Figure 1. A plot of the data collected from the Cygnus OB2 region. The survey area of the X-ray observations of Wright \& Drake (2009) is outlined by a grey dashed line. Each star is represented by a dot with a line. The position of the dot indicates the position of the star in RA ( $x$-axis) and Dec ( $y$-axis). The length and direction of the line coming from each dot indicates that star's velocity vector. Stars which are removed from the sample (as discussed in section 2.1) are shown by grey dots and vectors. In the top right hand corner is a $10 \mathrm{mas} \mathrm{yr}^{-1}$ (equal to $66 \mathrm{~km} \mathrm{~s}^{-1}$ at $1.4 \mathrm{kpc}$ ) vector with a grey cone outlining the median velocity uncertainty for the dataset.

\section{RESULTS}

\subsection{Magnitude definition: $\Delta v_{\mathrm{M}}$}

The VSAT method is applied to the dataset using the magnitude definition $\Delta v_{\mathrm{M}}$. The results are shown by the blue line in Fig. 5. On the $x$-axis of this figure is the distance between stars in parsecs, $\Delta r$, and on the $y$-axis is the average magnitude of the difference between stellar velocity vectors, $\Delta v_{\mathrm{M}}$, in $\mathrm{km} \mathrm{s}^{-1}$.
To demonstrate the degree of statistical noise the velocity vectors are randomly swapped between stars to remove any velocity structure. The method is re-run and the results recorded. This is done 1000 times. The area containing the central $1 \sigma$ of results is shown by a shaded grey region in Fig. 5. Features in the non-randomised results (blue line) on scales smaller than the spread of these randomised results (grey area) are not significant as they are within the fluctu- 


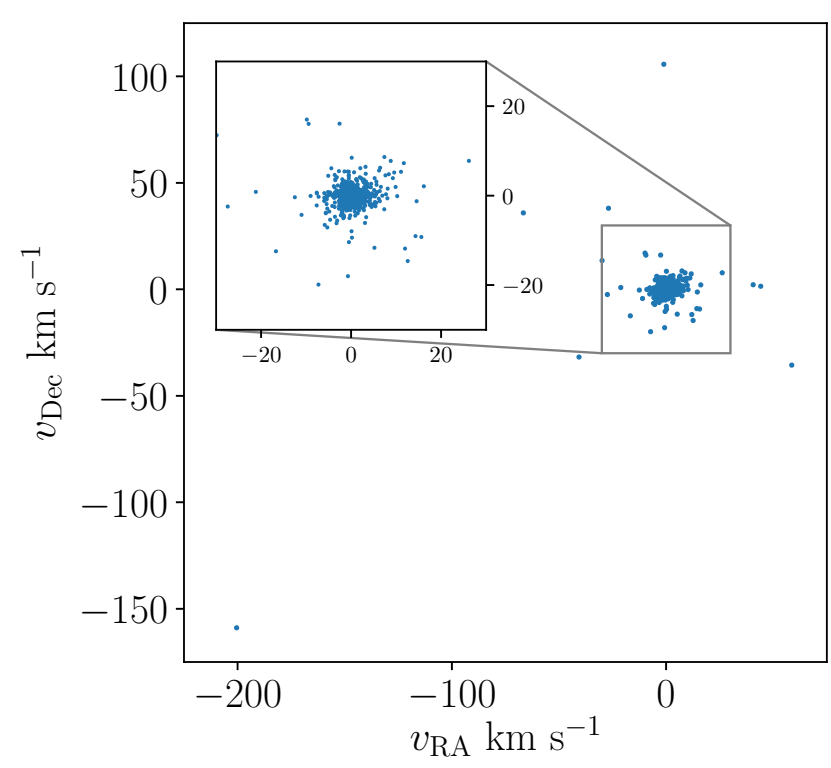

Figure 2. The RA and Dec stellar velocities plotted against one another.

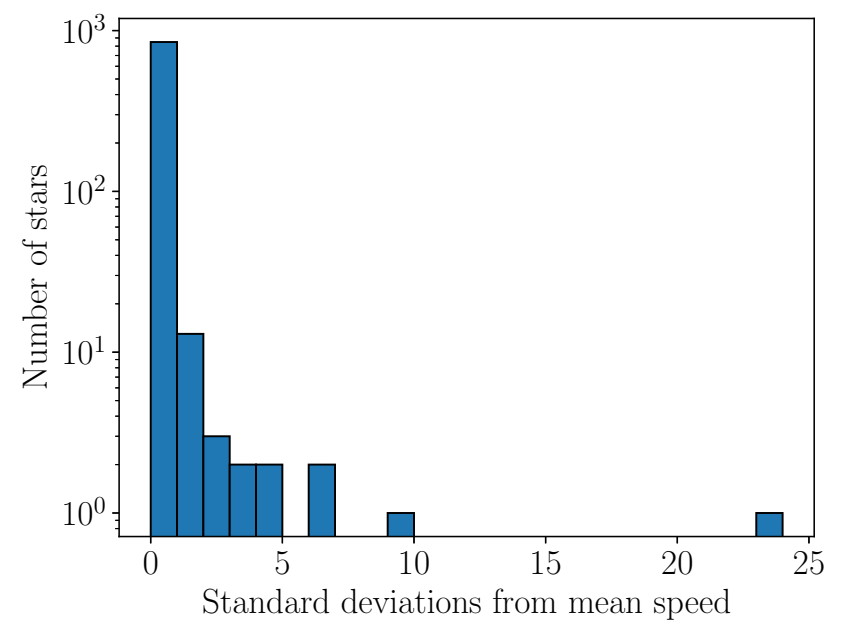

Figure 3. A histogram of the number of standard deviations stellar speeds are from the mean stellar speed in the Cygnus OB2 dataset. On the $x$-axis is number of standard deviations away from the mean. On the $y$-axis is the number of stars in each bin of the histogram. In order to ensure bins with a small number entries remain clearly visible the $y$-axis is logarithmic.

ations in measured velocity structure due to pure stochasticity.

Inspection of Fig. 5 shows a significant dip at scales $<0.5$ parsecs $^{1}$. This means that stars closer to each other

1 This feature is not visible if stars that are sigma clipped in section 2.1 are included. This is because, due to their locations, these stars feature in a disproportionately large number of low $\Delta r$ pairs, and the $<0.5$ parsec scale is particularly badly impacted. The sigma clipped stars have very different velocities to the rest of the dataset so their over representation causes an artificial in-

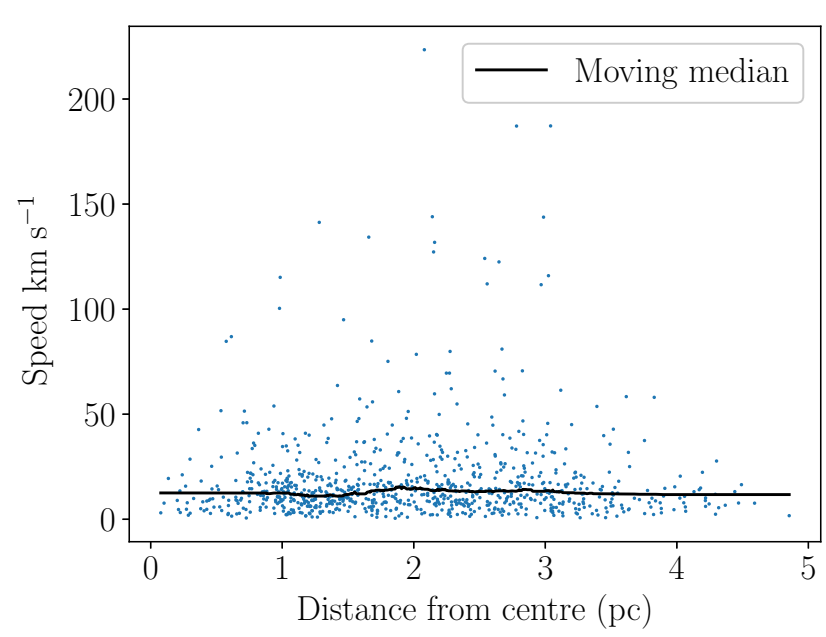

Figure 4. A scatterplot of stellar speeds plotted against their distance from the centre of the region. The moving median for this data is shown by a black line.

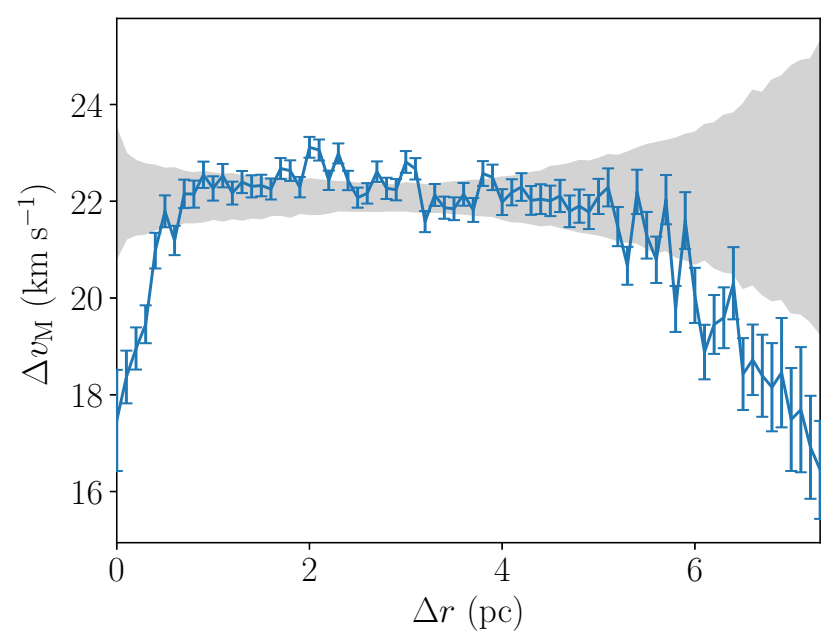

Figure 5. The velocity structure of Cygnus OB2 as determined by the VSAT method (Arnold \& Goodwin 2019) (blue line). The area containing the central $1 \sigma$ of the results of 1000 randomised cases is shown in grey. The $x$-axis shows the separation between stars in parsecs $\Delta r$, and on the $y$-axis is the average velocity difference of stellar pairs as defined by the magnitude definition of velocity difference, $\Delta v_{\mathrm{M}}$, in $\mathrm{km} \mathrm{s}^{-1}$.

than this tend to have similar velocity vectors relative to the velocities across the whole region studied.

Between $\sim 0.5$ - 5.5 parsecs the measured velocity structure (blue) is almost completely flat; there is no change in the magnitude of the difference between stellar velocity vectors as a function of how far apart they are. Further, the small fluctuations from flatness that are observed are almost entirely within the $1 \sigma$ bounds of those resulting from the regions where velocity structure has been removed by random

crease in $\Delta v_{\mathrm{M}}$. This explains their impact on the $<0.5$ parsec feature. 
shuffling (grey area). Therefore we conclude that there does not appear to be any velocity structure at these scales.

For $\Delta r>5.5$ parsecs $\Delta v_{\mathrm{M}}$ dips again. However, the results at these large scales are noisy and have high uncertainties. This is largely due to the fact that only stars on the outermost edges of the dataset are far enough apart to have such high $\Delta r$. Because of the morphology of the region which has lower stellar density towards its outskirts this means that there are fewer stellar pairs to populate these bins at large separations, and the results at $\Delta r \gtrsim 5.5$ parsecs are highly dependent on the exact velocity vectors of a small number of stars. Therefore although the measured 'structure' exceeds the bounds of the results with randomised velocity structures we cannot confidently determine whether velocity structure is or is not present at $\Delta r$ beyond 5.5 parsecs.

\subsection{Directional definition: $\Delta v_{\mathrm{D}}$}

The VSAT method is now applied using the directional definition of the velocity difference, $\Delta v_{\mathrm{D}}$. Recall that in this definition the more rapidly stars tend to move away from each other the more positive $\Delta v_{\mathrm{D}}$ is, and the more rapidly stars tend to move towards each other the more negative it is. The results are shown by the blue line in Fig. $6^{2}$. As in Fig. 5 the $x$-axis shows distance between pairs of stars, $\Delta r$, in parsecs. The directional velocity difference $\Delta v_{\mathrm{D}}$ is given on the $y$-axis in $\mathrm{km} \mathrm{s}^{-1}$. As in section 3.1 velocity vectors are then randomly shuffled between stars to remove any velocity structure, and the VSAT method is re-applied. This is done 1000 times. The $1 \sigma$ boundary of the results is plotted in grey on Fig. 6. This is done to give an idea of the amplitude of apparent velocity structures which in fact result from statistical noise.

The velocity structure of the region as measured using the directional definition of velocity difference does not fluctuate a great deal as a function of $\Delta r$. The blue line in Fig. 6 is largely flat, and the results are consistent with $\Delta v_{\mathrm{D}}=0$ (no net expansion or contraction) for $\Delta r<3.5$ parsecs. For $\Delta r$ between 3.5 and 6 parsecs $\Delta v_{\mathrm{D}}$ is only very slightly above 0 , and stays almost entirely within the bounds of the randomised cases with no velocity structure.

At $\Delta r>6$ parsecs the results show a positive correlation between $\Delta r$ and $\Delta v_{\mathrm{D}}$. Only stars on opposite sides of the dataset and close to its edges are far enough apart to populate these high $\Delta r$ bins. Additionally, as stated, positive $\Delta v_{\mathrm{D}}$ indicates a stellar pair is moving apart. Therefore this result implies that stars towards the fringes of the dataset are moving outwards, i.e that the region is expanding from it edges. However, for reasons discussed in section 3.1 VSAT is not reliable beyond $\Delta r=5.5$ parsecs for this dataset because of low number statistics. Therefore an additional test is conducted to verify whether this apparent expansion is real.

2 These results are virtually indistinguishable from the results if the eight stars removed in section 2.1 are included. The only impact is minor changes to the exact fluctuations of the lines in Fig. 6, but the same overall trend is observed. This is as expected: if the clipped stars are truly not members of Cygnus OB2 they should have more or less random directions, so no net impact on the results of the directional definition $\Delta v_{\mathrm{D}}$.

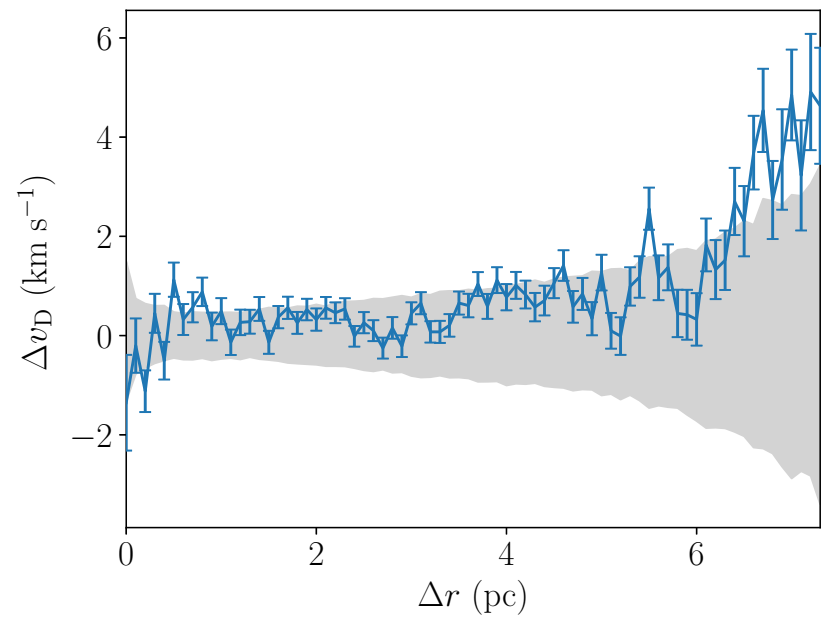

Figure 6. The velocity structure of Cygnus OB2 as determined by the VSAT method (Arnold \& Goodwin 2019) (blue line). The area containing the central $1 \sigma$ of the results of 1000 randomised cases is shown in grey. The $x$-axis shows the separation between stars in parsecs $\Delta r$, and on the $y$-axis is the average velocity difference of stellar pairs as defined by the directional definition of velocity difference, $\Delta v_{\mathrm{D}}$, in $\mathrm{km} \mathrm{s}^{-1}$.

First all the stars that are present in $\Delta r>6$ parsec bins (we will call these fringe stars) are identified, and how many times they appear in those bins is recorded. Next the centre of the dataset is determined by taking the average position of all stars. The direction of each fringe star's velocity relative to the centre of the dataset is then calculated, with $0^{\circ}$ indicating the star is moving radially outwards, $90^{\circ}$ that it is moving tangentially, and $180^{\circ}$ that is moving radially inwards. If fringe stars are moving systematically outwards then we expect their mean angle to be $<90 \pm 2.90^{\circ}$. Note $2.90^{\circ}$ is the expected standard deviation of the mean of a uniform distribution between 0 and $180^{\circ}$ given the number of datapoints.

The mean angle of the fringe star's velocity directions is calculated. This mean is weighted by how many times each star appears in $\Delta r>6$ parsec bins, as recorded earlier. The mean is $88.00^{\circ}$, however this is within the expected standard deviation of $2.90^{\circ}$ so therefore is not significant. From this we conclude there is no evidence of expansion or contraction at any scale in Cygnus OB2.

We emphasise that these findings do not constitute a null result. The fact that the data does not convincingly deviate from the range occupied by the randomised cases in Fig. 6 demonstrates that if there is any velocity structure in the dataset according to the $\Delta v_{\mathrm{D}}$ definition then it is so small as to be indistinguishable from random noise. As such the principle finding of this section is that if there is any velocity structure present in Cygnus OB2 is is less significant than random fluctuations.

This raises the question of how significant would the region's expansion have to be in order to be distinguishable from random fluctuations. In order to test this regions with increasingly significant expansion are simulated, and VSAT is applied. The regions are simulated by first drawing random positions from a uniform distribution and veloci- 
ties from a Gaussian distribution with a width of 1 , both centred on zero. Note the artificial regions are generated with the same number of stars as the Cygnus OB2 dataset. Random radial velocity components are then added to each star. The magnitudes of these velocities are also drawn from a Gaussian with a width of 1 and a mean of $R_{\text {sig }}$, which we define as the significance of the outwards expansion. This can be thought of as the ratio of systematic expansion to randomness. Note that if the number drawn for the radial magnitude is negative the radial component added will point inwards rather than outwards.

$R_{\text {sig }}$ is increased in increments of 0.01 . For each $R_{\text {sig }} 100$ regions are generated, VSAT is applied, and their $\Delta v_{\mathrm{D}}(\Delta r)$ is recorded. Results at $\Delta r>1$ are excluded as they rely on the 'corners' of the uniform distribution, and so rely on a smaller number of datapoints than results at smaller $\Delta r$ so are less reliable. This is analogous to the discounting of results at $\Delta r>5.5$ parsecs in the Cygnus OB2 dataset's results.

The $\Delta v_{\mathrm{D}}(\Delta r) \mathrm{s}$ of the expanding regions are then compared to simulated regions that are not expanding in order to determine if VSAT can differentiate them; another 100 regions that do not have systematic expansion are generated and VSAT is applied. For each $\Delta r$ the range of $\Delta v_{\mathrm{D}}$ s that contain the central $1 \sigma$ of results is recorded. These limits encapsulate the expected $1 \sigma$ fluctuation in $\Delta v_{\mathrm{D}}(\Delta r)$ due to stochasticity (analogous to the grey regions in Fig. 5 and Fig. 6). The mean fraction of $\Delta v_{\mathrm{D}}(\Delta r)$ datapoints outside of these limits for the expanding regions is recorded. As expected this fraction increases with $R_{\text {sig }}$, and increases beyond $1 \sigma$ significance when $R_{\text {sig }}$ is 0.07 . Therefore VSAT can identify expanding regions if the ratio of systematic expansion to random velocity dispersion is $\gtrsim 0.07$.

For reference a simulated region with $R_{\text {sig }}=0.07$ is shown in Fig. 7. By eye the expanding nature of this region can not be readily observed. This again demonstrates the need for quantitative techniques to analyse velocity substructure in star forming regions.

As will be discussed further in section 4.3 the velocity dispersion of Cygnus OB2 is difficult to characterise but it is likely of order $10 \mathrm{~s}$ of $\mathrm{km} \mathrm{s}^{-1}$. Wright et al. (2016) finds a velocity dispersion of $17.8 \mathrm{~km} \mathrm{~s}^{-1}$, assuming this is correct VSAT should be able to distinguish expansion $\geqslant 1.2 \mathrm{~km}$ $\mathrm{s}^{-1}$, therefore any systematic expansion in the region must be below this threshold.

\section{DISCUSSION}

\subsection{Discussion of results using $\Delta v_{M}$}

The results of our VSAT analysis of this part of Cygnus OB2 can be summarised as follows:

- there is velocity structure on scales $<0.5$ parsecs;

- scales $>0.5$ parsecs and $<5.5$ parsecs are consistent with a random velocity field;

- reliable conclusions cannot be drawn at scales > 5.5 parsecs.

In discussing these results it is also important to recall that the velocity dispersion of the data is of order $10 \mathrm{~s}$ of $\mathrm{km}$ $\mathrm{s}^{-1}$, the size of the field of view is of order 10 parsecs, and the age of the region is of order a few Myrs (Massey et al.
1995; Wright et al. 2015). As such is is likely the majority of the stars in the dataset did not form in this field of view but moved here from their birth sites of order 10s of parsecs away, erasing their original velocity structure. In this context the consistency of the results with a random velocity field is to be expected as the distances between the stars relative to one another represents a very small fraction of the total distances the stars have traveled from their origins.

Nevertheless there is velocity structure on scales $<0.5$ parsecs. This structure must either be primordial or have developed over time. It is extremely difficult to see how such structures could be constructed in a complex, unbound system such as a star forming region, so it is reasonable to assume these structures are primordial, i.e. this signature is due to probably fairly low- $N$ groups ${ }^{3}$ of stars that formed near each other and so with similar velocities according to the hierarchical paradigm of star formation (Elmegreen 2000). We can also conclude these groups must be bound as in order for unbound stars with similar velocities to remain within around 0.5 parsecs of each other for at least $3 \mathrm{Myr}$ (the approximate age of the region) the would have needed to form with a velocity dispersion of $\lesssim 0.17 \mathrm{~km} \mathrm{~s}^{-1}$.

To test this the local (within $0.5 \mathrm{pc}$ ) velocity dispersion of stars that are overrepresented in $<0.5 \mathrm{pc}$ bins are compared to the velocity dispersion of those that are not and are found to be a factor of 2.09 smaller on average. This provides further evidence of kinematic groups on 0.5 pc scales. However the velocity dispersions of stars overrepresented in $<0.5 \mathrm{pc}$ bins are many times in excess of $0.17 \mathrm{~km}$ $\mathrm{s}^{-1}$, indicating the groups must be bound in order to have remained so closely spatially associated. That said, it is likely that some stars have become unbound from the groups since they formed due to dynamical interactions, and that these groups formed with somewhat higher $N$ and larger size. However due to the youth of this region it is unlikely that the $N$ and size of these groups has changed a great deal since their formation.

Observationally the hypothesis of primordial bound groups with sizes of order $0.5-1$ parsecs is supported by the fact this scale is the typical size of star clusters (see e.g. Portegies Zwart, McMillan \& Gieles (2010)), the size of a typical molecular clump (Beuther et al. 2007), and is seen locally e.g. in Taurus (Gomez et al. 1993). A largescale random velocity field with many 'embedded' $\sim 0.5$ parsec bound groups is also consistent with the high degree of spatial structure found by Wright et al. (2014) (they measure the Cartwright \& Whitworth (2004) $Q$-parameter to be 0.4-0.5). It also fits the finding of Griffiths, Goodwin \& Caballero-Nieves (2018) that the number of wide massive binaries in Cygnus OB2 suggests many (at least 30) different sites of massive star formation across the entire association.

\subsection{Discussion of results using $\Delta v_{\mathrm{D}}$}

As discussed in section 3.2 the velocity structure as measured using the directional definition of velocity difference is very flat. $\Delta v_{\mathrm{D}}$ is consistent with 0 for $\Delta r<3.5$ parsecs, indicating the region is neither expanding or contracting on

${ }^{3}$ It is not clear to us if they deserve the title 'clusters' 


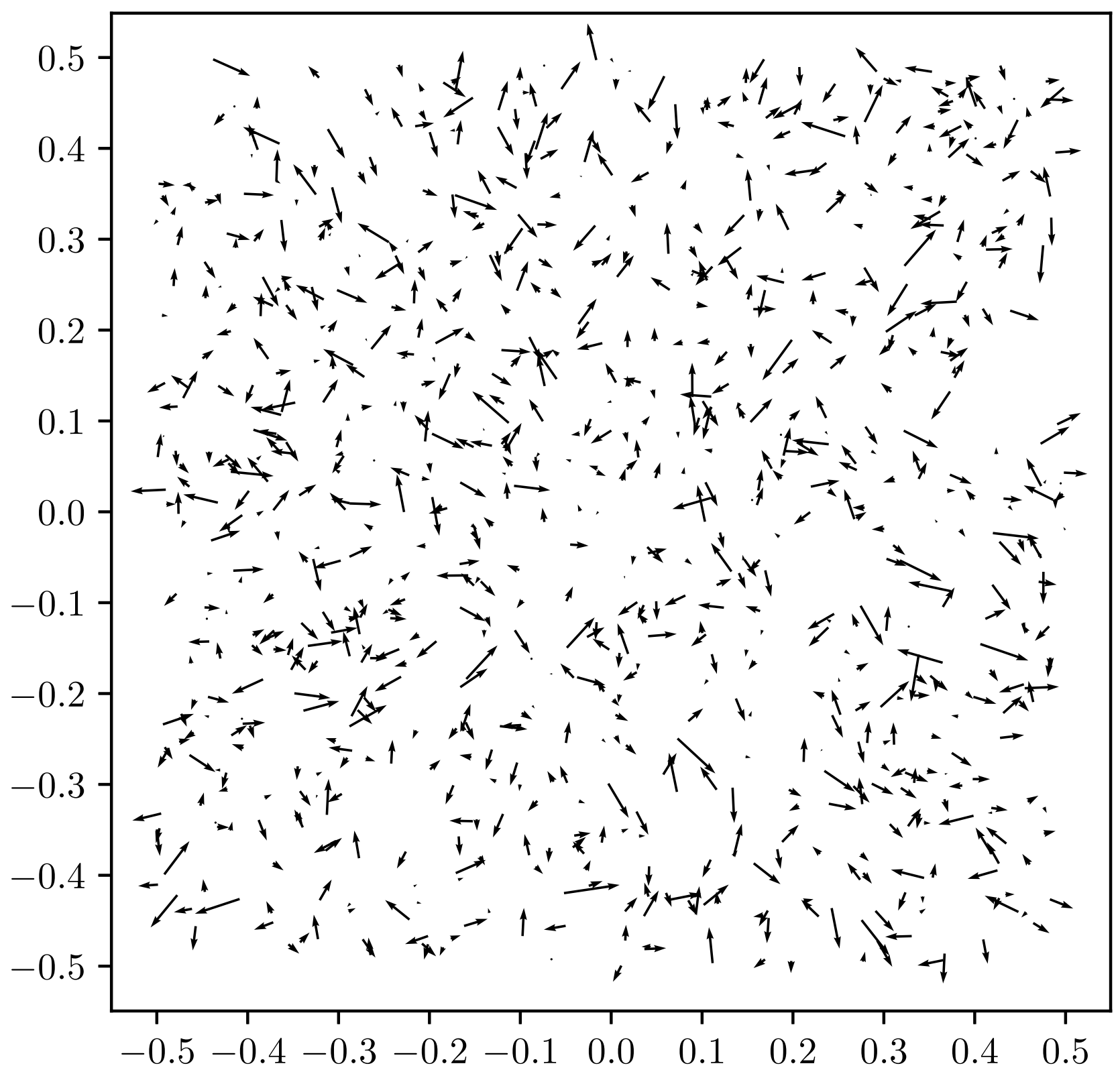

Figure 7. A simulated region with an $R_{\text {sig }}$ of 0.07 . Each star is shown as an arrow where the length and direction of the arrow reflects the star's velocity, and its position is the position of the star.

these scales. Further, for $\Delta r$ between 3.5 and 6 parsecs fluctuations in $\Delta v_{\mathrm{D}}$ are within the bounds of the cases where any systematic velocity structure is removed by random shuffling of the velocity vectors between stars (grey area in Fig. 6). As such we conclude that any systematic expansion or contraction occurring within the region at scales $<6$ parsecs is less significant than random noise. Further VSAT is able to distinguish systematic expansion/contraction from random noise if the ratio of the systematic component to the velocity dispersion of the region is $\gtrsim 0.07$, so if any exists it must be very small.

At larger $\Delta r$ there is a positive correlation between $\Delta v_{\mathrm{D}}$ and $\Delta r$. This indicates that at these scales stars further away from each other tend to be moving apart, i.e. the region may be expanding from the edges. However the VSAT method is not reliable at such large $\Delta r$ because of small number statistics so an additional statistical test is conducted and no expansion is found.

As a result we conclude from Fig. 6 that there is no evidence of systematic expansion or contraction on any scale in Cygnus OB2, and if any exists it is far less significant than random noise. This makes sense if, as we argue in section 4.1, the stars in this dataset formed across the Cygnus star forming complex and happen to be in this field of view at the 
time of observation. If this is the case we would not expect the stars to move in systematic directions relative to one another, and indeed directional structure is not observed in Fig. 6.

\subsection{Comparison of results to other kinematic studies of Cygnus OB2}

As has been mentioned Cygnus OB2 has been the subject of a number of other kinematic studies. Here we compare our results to the findings of Wright et al. (2014), Wright et al. (2016), and Winter et al. (2019).

In section 3.1 we find evidence of kinematic substructure at scales $\leqslant 0.5 \mathrm{pc}$. In agreement with this Wright et al. (2016) applies a number of statistics which confirm the presence of kinematic substructure in the region. However from a by-eye inspection of the data Wright et al. (2016) asserts that kinematic substructure is present at a range of scales which is not consistent with our results. From the results of section 3.1 we argue that Cygnus OB2 formed with a hierarchical, substructured morphology. This is in agreement with Winter et al. (2019) which uses $N$-body simulations and observations of the properties of protoplanetary disks in Cygnus OB2 to constrain the initial conditions of the region, and finds the region must have formed with substructure to reproduce the observations. Specifically Winter, Clarke \& Rosotti (2019) finds Cygnus OB2 may originate from a superposition of multiple clusters, and their Figure 8(a) looks to the eye to be moderately similar to our Fig. 1. However, it might well lack the 0.5 parsec structures we find in the real data (as that scale was not imposed in their initial conditions and it is difficult to see how it could arise dynamically). A possible avenue for future work could be to analyse a variety of different idealised initial conditions to see which best-fit the real data.

We do a simple analysis and find the velocity dispersion of the region to be $28.72 \pm 0.62 \mathrm{~km} \mathrm{~s}^{-1}$ which is not consistent with the result found by Wright et al. (2016) which is $17.8 \pm 0.6 \mathrm{~km} \mathrm{~s}^{-1}$. This disagreement is explained by the fact the datast used here covers a larger region than Wright et al. (2016). This discrepancy notwithstanding in both cases the region is found to be unbound. Additionally Winter et al. (2019) (which also looks at a larger region than Wright et al. (2016)) estimated the initial velocity dispersion of the region to be $50 \mathrm{~km} \mathrm{~s}^{-1}$.

We conclude that the velocity dispersion is relatively high and is likely of order $10 \mathrm{~s}$ of $\mathrm{km} \mathrm{s}^{-1}$. Given this and the region's age we conclude some 'mixing' should have had time to occur, erasing velocity substructure on scales that are not bound. This fits uncomfortably with the assertion of Wright et al. (2016) that the region is not dynamically evolved. We argue that given the relatively low-density nature of Cygnus OB2 that we would not expect stars to have had a very large number of dynamical interactions despite traveling potentially large distances. Nevertheless we expect some degree of dynamical evolution to have occurred.

In Wright et al. (2016) the total kinetic energy of stars moving radially inwards and outwards from the region are compared and found to be nearly identical (the ratio is 51 per cent to 49 per cent). From this it is concluded that there is no net expansion or contraction in Cygnus OB2. This result is called into question by Winter et al. (2019) which argues that this ratio does not provide a good measure of whether a region is expanding or contracting. However in this paper, using an entirely different method, we also find that there is no evidence of expansion or contraction in Cygnus OB2. This result suggests that the region did not evolve to its current large, diffuse structure by expanding from a significantly denser one as would be expected by some models (Lada et al. 1991) (Carpenter 2000).

This suggestion is in agreement with the conclusions drawn in Wright et al. (2014) from their analysis of Cygnus OB2's spatial structure. Despite its consistency with previous results this finding is still odd. The Cygnus OB2 region has a velocity dispersion of $10 \mathrm{~s}$ of $\mathrm{km} \mathrm{s}^{-1}$ and, given its estimated mass of $\sim 10^{4} M_{\odot}$, it should be supervirial. Conventional logic states it should be expanding rapidly, but it does not appear to be doing so. However from its studies of protoplanetary disk properties Winter et al. (2019) estimates that gas removal ceased just 0.5 Myr ago and the region was not originally unbound. It is conceivable that there has been insufficient time for the region to 'feel the effects' of this change. This could explain why it is not noticeably expanding yet, but may begin to do so in the near future. This would be consistent with the conclusion drawn in Wright et al. (2016) that within 4 Myr Cygnus OB2 will expand to be over $100 \mathrm{pc}$ in size.

\section{CONCLUSIONS}

We analyse the positions and proper motions of stars in a region of Cygnus OB2 from Wright et al. (2014) using the VSAT method presented in Arnold \& Goodwin (2019).

Our main findings are:

- Stars within 0.5 parsecs of each other have significantly similar velocities.

- At all reliable scales larger than 0.5 parsecs velocities are consistent with a random distribution.

- We find no evidence of systematic expansion or collapse in this part of Cygnus OB2. If any such systematic motion exists its significance is $\lesssim$ a factor of 0.07 of that of the velocity dispersion of the region, and cannot be distinguished from random noise by the methods employed in this paper.

This suggests that we are observing many primordial bound structures on scales $<0.5$ parsecs ('groups' or small 'clusters'). However, any initial velocity structure on scales larger than 0.5 parsecs has been erased by stars having moved 10s of parsecs in the few Myr since they formed. Within this region we see no significant evidence for global expansion or contraction (but we note this is only the central part of Cygnus OB2).

\section{ACKNOWLEDGEMENTS}

NJW acknowledges an STFC Ernest Rutherford Fellowship (grant number ST/M005569/1). BA acknowledges PhD funding from the University of Sheffield. Many thanks also to Stuart Littlefair for useful discussions and insights, and the referee for helpful comments and suggestions which improved this work. 


\section{REFERENCES}

Allison R. J., Goodwin S. P., Parker R. J., Portegies Zwart S. F., de Grijs R., Kouwenhoven M. B. N., 2009, MNRAS, 395, 1449

Arenou F., et al., 2018, A\&A, 616, A17

Arnold B., Goodwin S. P., 2019, MNRAS, 483, 3894

Berlanas S. R., Herrero A., Comerón F., Simón-Díaz S., Cerviño M., Pasquali A., 2018, A\&A, 620, A56

Berlanas S. R., Wright N. J., Herrero A., Drew J. E., Lennon D. J., 2019, MNRAS, 484, 1838

Beuther H., Churchwell E. B., McKee C. F., Tan J. C., 2007, in Reipurth B., Jewitt D., Keil K., eds, Protostars and Planets V. p. 165 (arXiv:astro-ph/0602012)

Bouy H., Bertin E., Moraux E., Cuillandre J. C., Bouvier J., Barrado D., Solano E., Bayo A., 2013, A\&A, 554, A101

Buckner A. S. M., et al., 2019, A\&A, 622, A184

Carpenter J. M., 2000, AJ, 120, 3139

Cartwright A., 2009, MNRAS, 400, 1427

Cartwright A., Whitworth A. P., 2004, MNRAS, 348, 589

Comerón F., Pasquali A., Figueras F., Torra J., 2008, A\&A, 486, 453

Drew J. E., Greimel R., Irwin M. J., Sale S. E., 2008, MNRAS, 386, 1761

Elmegreen B. G., 2000, ApJ, 530, 277

Gomez M., Hartmann L., Kenyon S. J., Hewett R., 1993, AJ, 105, 1927

Griffiths D. W., Goodwin S. P., Caballero-Nieves S. M., 2018, MNRAS, 476, 2493

Hanson M. M., 2003, ApJ, 597, 957

Kiminki D. C., Kobulnicky H. A., Vargas Álvarez C. A., Alexander M. J., Lundquist M. J., 2015, ApJ, 811, 85

Knödlseder J., 2000, A\&A, 360, 539

Lada E. A., Depoy D. L., Evans Neal J. I., Gatley I., 1991, ApJ, 371,171

Lindegren L., 2018, in Recio-Blanco A., de Laverny P., Brown A. G. A., Prusti T., eds, IAU Symposium Vol. 330, Astrometry and Astrophysics in the Gaia Sky. pp 41-48, doi:10.1017/S1743921317005919

Lindegren L., et al., 2018, A\&A, 616, A2

Maschberger T., Clarke C. J., 2011, MNRAS, 416, 541

Massey P., Thompson A. B., 1991, ApJ, 101, 1408

Massey P., Johnson K. E., Degioia-Eastwood K., 1995, ApJ, 454, 151

Portegies Zwart S. F., McMillan S. L. W., Gieles M., 2010, ARAA, 48,431

Roquette J., Bouvier J., Alencar S. H. P., Vaz L. P. R., Guarcello M. G., 2017, A\&A, 603, A106

Rygl K. L. J., et al., 2012, A\&A, 539, A79

Winter A. J., Clarke C. J., Rosotti G. P., 2019, MNRAS, 485, 1489

Wright N. J., Drake J. J., 2009, ApJS, 184, 84

Wright N. J., Drake J. J., Drew J. E., Vink J. S., 2010, ApJ, 713, 871

Wright N. J., Parker R. J., Goodwin S. P., Drake J. J., 2014, MNRAS, 438, 639

Wright N. J., Drew J. E., Mohr-Smith M., 2015, Monthly Notices of the Royal Astronomical Society, 449, 741

Wright N. J., Bouy H., Drew J. E., Sarro L. M., Bertin E., Cuillandre J.-C., Barrado D., 2016, MNRAS, 460, 2593

This paper has been typeset from a $\mathrm{TEX}_{\mathrm{E}} / \mathrm{LAT}_{\mathrm{E}} \mathrm{X}$ file prepared by the author. 